\title{
Parasite Ovum Automatic Recognition in the High Noise of Microscopic Image
}

\author{
Fengshou Zhang ${ }^{1}$, Xin Meng ${ }^{1, *}$, Zhigang $\mathrm{Hu}^{2}$ and Siwen $\mathrm{Li}^{1}$ \\ ${ }^{1}$ Henan university of science and technology, Mechanical and electrical engineering college, luoYang, HeNan, China \\ ${ }^{2}$ Henan university of science and technology, Medical technology and engineering college, luoYang,HeNan, China \\ *Corresponding author
}

\begin{abstract}
An automatic recognition method of parasitic ovum under the high noise is studied in this thesis. Considering the status of the complex background in the parasitic ovum images, firstly, the corresponding method is applied to image preprocessing, then, the noise removing and edge segmentation combined with threshold segmentation and mathematical morphology operation are emphatically studied, then feature extraction and parasitic ovum classification are processed, gaining a set of algorithm process to recognize parasitic ovum in complex background rapidly and accurately. Finally, this method is tested by the recognition experiments of three kinds of parasitic ovum, the results verify the effectiveness of the method.
\end{abstract}

Keywords-parasite ovum; threshold segmentation; mathematical morphology; feature extraction; BP neural network; automatic identification

\section{INTRODUCTION}

In recent years, with the development of computer technology, image processing and artificial intelligence have been rapid development. The automatic identification study of parasitic ovum combined with digital image processing and mode recognition has been the emerging direction ${ }^{[1]}$. For example, Xiao-min GUO, Xiao-ming $\mathrm{WU}^{[2]}$ designed a classifier based on probabilistic neural network, by 9 kinds of parasitic ovum for automatic identification, obtained a better result, which the average correct recognition rate is $99.23 \%$. Li Feng, Qi-yan SUN ${ }^{[3]}$ put forward a parasitic ovum's classification algorithm, which based on the edge of space distribution histogram, experiments show that the method has a good recognition rate and operating efficiency. Di WANG ${ }^{[4]}$ realized parasitic ovum's automatic identification by using linear discriminant analysis, experiments show that this method is feasible, which the correct recognition rate reaches $90.70 \%$. But they are not to analysis and explanation the noise removal and image segmentation method of parasitic ovum digital image in-depth. In view of parasitic ovum image's characteristics of high noise and complex background, this paper mainly studied the parasitic ovum digital image's noise removal and segmentation, and put forward a method based on threshold segmentation combined with morphological operation, which can completely apart from the noise and edge segmentation, finally realized the parasitic ovum recognition classification by using the BP neural network error training method. Automatic identification process is shown in Figure I.

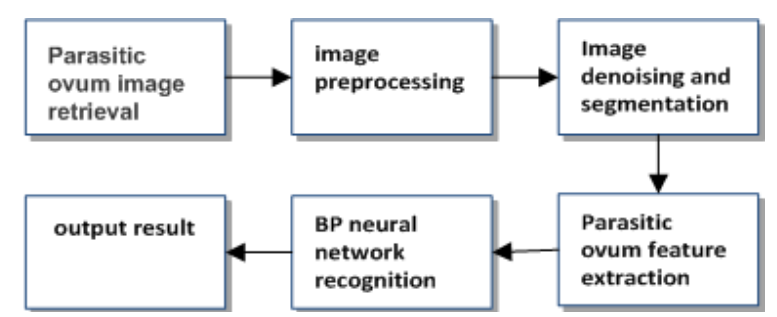

FIGURE I. THE BASIC FLOW SHEET OF PARASITIC OVUM'S AUTOMATIC IDENTIFICATION

\section{PARASITIC OvUM IMAge PREPROCESSING AND SEGMENTATION}

\section{A. Parasitic Ovum Image Preprocessing}

In the process of parasitic ovum microscopic image acquisition, images will appear some random high frequency noise because of the equipment, in addition, parasitic ovum image itself contains a lot of noise, which far more than the external noise. If completely removing the noise in the preprocessing stage, the parasitic ovum's outline will be serious deformation and loses its characteristics. Through repeated experiments, this article only do $3 \times 3$ median filter in the preprocessing stage, the purpose is to remove the salt and pepper noise in the images, improve image's quality, and get prepare for noise removing completely combined with threshold segmentation and mathematical morphology operation in the next section. The original image are shown in Figure II, Preprocessing results are shown in Figure III .

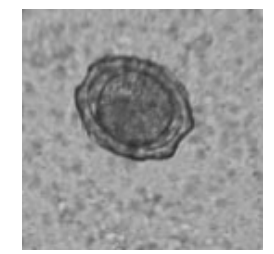

(a)ova of roundworm

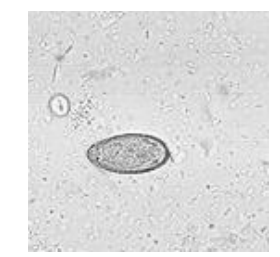

(b)ova of liver fluke

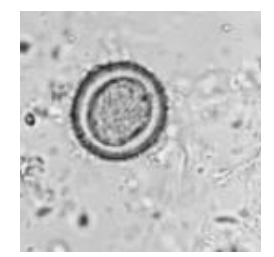

(c)ova of tapeworm
FIGURE II. PARASITIC OVUM ORIGINAL

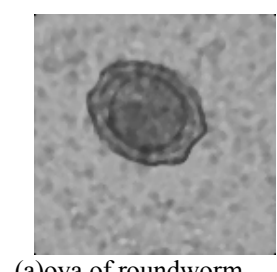

(a)ova of roundworm

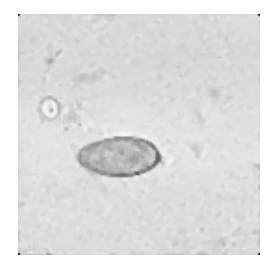

(b)ova of liver fluke

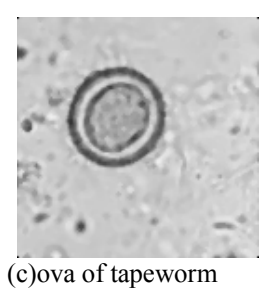

(c)ova of tapeworm
FIGURE III. PARASITIC OVUM AFTER PRETREATMENT 
By the picture above, we can find that after median filtering, the salt and pepper noise be removed, and the quality of Image is improved, but there are still a lot of noise in the background, this will be removed in the subsequent processing.

\section{B. Parasitic Ovum Image Segmentation}

After preprocessing, then the image segmentation of parasitic ovum will be done, this is a key step from image processing to image analysis, this will have an important influence on feature extraction. Commonly used image segmentation methods are edge segmentation, threshold segmentation and region segmentation. Then, the shape and contour of the target are extracted by morphological method.

In this paper, many experiments are made according to the characteristics of the parasitic ovum images, finally this paper choose the threshold segmentation method (Otsu) combined with mathematical morphology operation, this method not only get rid of all the noise, but also got the precise contour of the target. Algorithm process is as follows:

(1) After pretreatment, using Otsu method to deal with the image for binarization processing;

(2) Select the appropriate structure elements to conduct the multiple closed operation to the binarization image, until the internal hole of parasitic ovum filling out.

(3) Select the appropriate structure elements to conduct the multiple open operation to the image, until all the noise filter out

\section{(4) Extract the image contour}

Then the algorithm will be explained in detail:

Otsu threshold segmentation method is deduced on the basis of decision analysis of least square method, this method is simple, is a stable and commonly used algorithm. using Otsu method to deal with the image is shown as Figure IV.

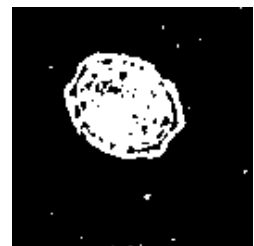

(a)ova of roundworm

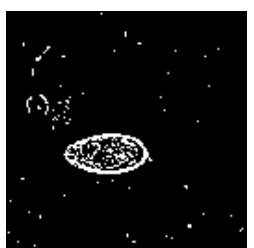

(b)ova of liver fluke

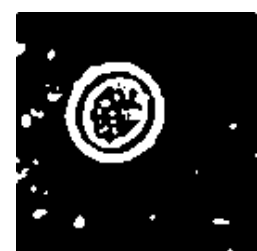

(c)ova of tapeworm
FIGURE IV. OSTU THRESHOLD SEGMENTATION

As shown in Figure IV, some of the noise is removed at the same time of binarization, but those noise whose gray value is closed to the target is still remaining in the image, the noise will be completely removed by morphological operations later on.

Morphological operation ${ }^{[5]}$ : After threshold segmentation, parasitic ovum images appeared breaking point and a lot of holes in itself because of the target pixel is not balanced. And because of the complex background of the parasitic ovum image, there are still a lot of small noise in the image after threshold segmentation, for this we need to connect and fill the binary image and filter out the noise at the same time, these operations can be completed by the method of morphology. In this paper, we do the Closed Operation first to the image after the threshold segmentation, then do the Open Operation.

Closed Operation is one of the basic operations of morphology. It is first expansion then corrosion to image, the expression is:

$$
\mathrm{A} \bullet \mathrm{S}=(\mathrm{A} \oplus \mathrm{S}) \Theta \mathrm{S}
$$

The result of the Closed Operation is to make the image contour smooth, it can usually fit the narrow gap, fill the small hole. Therefore, in this paper, the image was done Close Operation first, the purpose is to populate the hole in the target.

The Open Operation is opposite to closed operation, It is first corrosion then expansion to image, the expression is:

$$
\mathrm{A} \circ \mathrm{S}=(\mathrm{A} \Theta \mathrm{S}) \oplus \mathrm{S}
$$

The result of the Open Operation is to make the image boundary smoothing, and it can get rid of the area that is not contain structure information, disconnect the narrow connection, retain the object area that contains the structural elements, and keep the image's shape and size unchanged. Morphological operation effect as shown in Figure V.

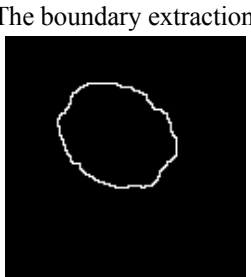

(a)ova of roundworm

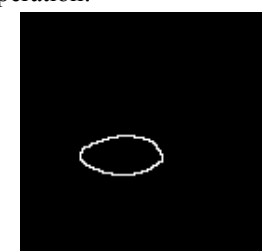

(b)ova of liver fluke

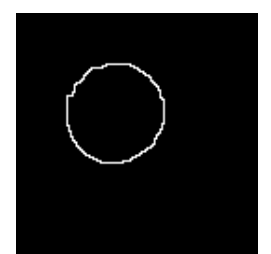

(c)ova of tapeworm
FIGURE V. MORPHOLOGICAL OPERATION

As we can see from above, after threshold segmentation and morphological processing, we not only successfully remove the noise, but also got the detailed binary image and contour of parasitic ovum, from which We can get the parasitic ovum morphological characteristics, this create a conditions for the next extraction of the characteristics.

\section{PARAsitic Ovum Image Feature Extraction}

When the parasitic ovum segmented from the background, the next step is to accurately extract the characteristics of the parasitic ovum, feature extraction will directly affect the classifier design and classification results ${ }^{[6]}$.

In this paper the classification of parasitic ovum, through the analysis and many experiments of the image, eventually pick up nine features from the three major features of image as the feature recognition of this article, they respectively are area $\mathrm{A}$, perimeter P, Circle C, Rectangular degree R, Elongation E, average gray-value ${ }^{\overline{\mathrm{r}}}$, gray variance ${ }^{\sigma^{2}}$, Texture contrast CON, texture entropy ENT.

After the selection of target feature vector, the next is the feature extraction of the target. The extraction of target feature vector in this article is based on Matlab software platform, 
through the gray image and binary image and the grey level histogram, we can obtained the area and perimeter, then according to the formula, we can get several other types of geometry features. Finally, all the characteristics that have extracted are given in Table I, all characteristic has carried on the normalized processing.

TABLE I. PARASITIC OVUM'S EIGENVALUES

\begin{tabular}{llll}
\hline \multicolumn{1}{c}{ feature code } & $\begin{array}{c}\text { ova of } \\
\text { roundworm }\end{array}$ & $\begin{array}{c}\text { ova of } \\
\text { liver fluke }\end{array}$ & $\begin{array}{c}\text { ova of } \\
\text { tapeworm }\end{array}$ \\
\hline & & & \\
Area A & 2755 & 758 & 2291 \\
Perimeter P & 252.82 & 153.07 & 210.00 \\
$\begin{array}{l}\text { Circle C } \\
\text { Rectangular degree R }\end{array}$ & 0.5452 & 0.4178 & 0.6528 \\
ElongationE & 0.7672 & 0.7324 & 0.8005 \\
& 0.9048 & 0.5111 & 0.9815 \\
average gray-value & $\bar{r}$ & & \\
& 92.27 & 200.36 & 160.35 \\
gray variance $^{2}$ & & & \\
Texture contrast CON & 10.23 & 12.33 & 14.58 \\
texture entropy ENT & 0.52 & 0.23 & 0.39 \\
& 2.19 & 0.83 & 1.52 \\
\hline
\end{tabular}

\section{IMAGE RECOGNITION OF PARASITIC OVUM}

After extracting the characteristics of parasitic ovum, the next step is to design a classifier, then the extracted features as the input vector put into the classifier to do the classification of parasitic ovum image. Through the contrast in this paper, BP neural network is finally chosen as the feature's classifier of parasitic ovum image.

Due to BP neural network structure is simple, highly nonlinear mapping ability, highly self-learning adaptive ability, and good fault tolerance and so on, it got a wide application ${ }^{[7]}$. The design of BP network mainly includes number of Layers, input layer, hidden layer and output layer node number, transmission function, training method, training parameter and so on. The BP neural network in this article is composed of input layer, hidden layer and output layer of three parts; the number of neurons in input layer was set to 9; for the hidden layer, finally determine the optimal number of neurons is 5 ; the number of output layer nodes is set to 3 . Finally we put the characteristics of parasitic ovum samples into BP neural network classifier for recognition. The training function of BP neural network classifier set as trainlm, then implement this with the aid of Matlab simulation software. This article selects three kinds of parasitic ovum as the training sample, the training process is as the following Figure VI.

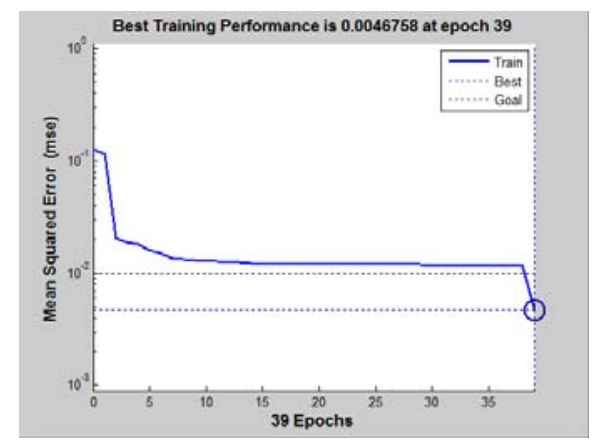

FIGURE VI. TRAINING RESULTS
After learning by training samples, then we can use the test sample to test the BP neural network classifier. Then we put three kinds of parasitic ovum that have been extracted into the BP neural network for identification and classification. Test results are given in Table II.

TABLE II. RESULTS OF BP NEURAL NETWORK TRAINING

\begin{tabular}{llll}
\hline \multicolumn{1}{c}{ parasitic ovum } & $\begin{array}{c}\text { Test-sample } \\
\text { number }\end{array}$ & $\begin{array}{c}\text { the correct } \\
\text { number }\end{array}$ & accuracy \\
\hline ova of & & 33 & $94 \%$ \\
roundworm & 35 & 36 & $90 \%$ \\
ova of liver fluke & 40 & 37 & $93 \%$ \\
ova of tapeworm & 40 & & \\
\hline
\end{tabular}

By the Table II, the identification of parasite eggs in more than $90 \%$, the accuracy of identification is able to achieve the desired effect, the automatic recognition method of parasitic ovum can meet the actual requirements.

\section{CONCLUSION}

Through the research and comparison of the parasitic ovum image In the high noise, this article effectively implemented the division of the parasitic ovum and contour extraction, then effectively extract the characteristics of the parasitic ovum on Matlab software platform, finally, BP neural network identifier is employed to identify, and obtain a higher identification accuracy.

\section{ACKNOWLEDGEMENTS}

Science and Technology Research Project of Henan Province(122102310276).

Production-study-research Cooperation Project of Henan Province (2015HNCXY003).

\section{REFERENCES}

[1] Sommer C Digtial image analysis and identification of eggs bovine parasitic nematodes[J].Journal of Helminthollogy 1996,70(2): 143- 151.

[2] Xiaomin GUO, Xiaoming WU,Ximei ZHAN.Recognition of Images of Parasite Egg Based on Probabilistic Neural Network[J] .Computer Engineering and Applications,2005.15:198-199.

[3] Feng LI, Qiyan SUN. Study on Image Shape Classification Algorithm of Human Parasite Eggs Based on Boundary Features[J] - Computer Science 2012,39(5)261-265.

[4] Di WANG.Parasite Eggs Recognition Based on Linear Discriminant Analysis[J].Modern Computer,2014,(5)18-22.

[5] Zheng ZHANG,Hongxia NI,Chunmiao YUAN.Digital Image Processing [M]. Posts and Telecom Press, 2016,225-232.

[6] Zongjing XU, Ping HU.Texture Feature Extraction Methods of Microgra ph Image. Microcomputer pplications.2009,30(6): 6-13.

[7] Yongjun CAI, Weiling LIU, Qilian YU.Application of genetic neuraI network to recognition of urine sediment. Chinese Medical Equipment Jo urnal,2004,25(11): 1-2. 